\title{
Identification Method for Conductor Measurement Temperature in Dynamic Capacity-Increase of Transmission Line
}

\author{
Peng Wang, 2, a, Shuhai Feng ${ }^{2, b}$, Yong Wang ${ }^{2, ~ c}$, Jun Liü, d, Jinquan Zhao ${ }^{1, \text { e }}$ \\ ${ }^{1}$ Hohai University, Nanjing 210098, China \\ ${ }^{2}$ China Electric Power Research Institute, Nanjing 210003, China \\ a jswpagb@foxmail.com, ${ }^{b}$ fengshuhai@epri.sgcc.com.cn, ${ }^{c}$ wangyong5@epri.sgcc.com.cn, \\ ${ }^{\mathrm{d}}$ liujun@epri.sgcc.com.cn, ${ }^{\mathrm{e}}$ zhaojinquan@hhu.edu.cn
}

Keywords: DTCR, Identification Method, Dynamic Capacity-Increase, Temperature.

Abstract. Dynamic capacity-increase of transmission line will be a practical solution to the rising demand for load. This paper proposes a method for identifying the temperature measurement value of transmission line on a continuous time section. The method is based on the study of the transient thermal stability equation of wire temperature. It focuses on the transient process of wire temperature changing with time and considers the cross section data. The time axis is used to identify the cross section data, which is a practical method with high identification accuracy. The method is verified by the experimental data of the dynamic capacity increasing demonstration project.

\section{Introduction}

Dynamic capacity-increase of transmission line is a practical solution to the rising demand for load [1, $2,3]$. This paper proposes a method for identifying the temperature measurement value of the wire on a continuous time section. The method is based on the study of the transient thermal stability equation of wire temperature. It focuses on the transient process of wire temperature changing with time and considers the cross section data. The time axis is used to identify the cross section data, which means the method has practical feasibility and high identification accuracy. In the end, the method is verified by the experimental data of the dynamic capacity increasing demonstration project, as follows.

The formula for calculating the current capacity of the transmission line is derived from the temperature rise of the wire heating and the balance of heat and cooling under standard state. The conductor and the external environment are always in the exchange of heat. When the temperature of the transmission line is stable at a certain value, the system can reach the stable equilibrium. The specific process is as follows:

When current is passed through the wire, electrical energy is converted into heat energy by wire resistance. A portion of the heat energy stored in the conductor and insulation material, and the remaining transferred to the surrounding material in the form of convection and radiation. At the same time, the conductor will absorb the heat generated by the sunshine radiation. And the heat causes the wire temperature rise, the dynamic process continues until the line temperature is in steady state.

So the equation describing the process is transient, and to the final result, it is stable [4] .

When electricity $\left(E_{i n}\right)$ flows through the wire, part of the $E_{\text {in }}$ is converted into heat energy $\left(Q_{I}\right)$, and the wire keeps absorbing sunlight energy $\left(Q_{S}\right)$ at the same time. $Q_{I}$ and $Q_{S}$ constitute the total heat of the conductor. At the same time, the heat is transferred to the surrounding material in the form of convection $\left(Q_{C}\right)$ and radiation $\left(Q_{R}\right)$ from the wire [5]. In the transient state, the amount of heat absorbed is greater than the heat dissipation, and the temperature of the wire is increased continuously until it reaches the steady state. At steady state, the heat absorbed and heat dissipation are balanced, and the temperature is kept constant. Transient formula is as Eq. $1[6,7]$.

$$
Q_{I}+Q_{S}=Q_{R}+Q_{C}+m C \frac{d T}{d t} \text {. }
$$


The simplified transient formula can be obtained to Eq. 2 [8] .

$$
\begin{aligned}
\frac{d T}{d t}=\frac{\alpha_{S} I_{S} D-I^{2} K R_{20}\left[1+\alpha_{20}(T-20)\right]}{m C}- & \frac{\pi \varepsilon S D\left[(T+273)^{4}-\left(t_{a}+273\right)^{4}\right]+9.92\left(T-t_{a}\right)(V D)^{0.485}}{m C} .
\end{aligned}
$$

And the steady state formula can be simplified as Eq. 3 [9] .

$$
I^{2}=\frac{\pi \varepsilon S D\left[(T+273)^{4}-\left(t_{a}+273\right)^{4}\right]+9.92\left(T-t_{a}\right)(V D)^{0.485}-\alpha_{S} I_{S} D}{K R_{20}\left[1+\alpha_{20}(T-20)\right]}
$$

\section{Identification Method of Measured Wire Temperature}

Two kinds of factors are considered, the vertical ones and the horizontal ones, to makes the identification result as accurate as possible. The vertical ones are the factors changing with time; the horizontal ones are the factors changing with location, specifically the data from different measurement devices at the same time. For the same line, the horizontal data is divided into broad and narrow horizontal data.

Broad horizontal data: measured data on different location of one same line, such as the wind speed data on tower A and the wind speed data on tower B are broad horizontal data.

Narrow horizontal data: measured data of different measuring devices on the same location, such as the wind speed data of device $a$ on tower A and the wind speed data of device $b$ on tower A are narrow horizontal data.

Regarding to the difference between the micro meteorological monitoring data and wire temperature measurement values on different location, the broad horizontal data of one line has great difference (especially wind speed and wind direction data). In this paper, the main idea is to use the vertical data and narrow horizontal data. So we just focus on the data of one tower during the process of identification.

Then, we will introduce how to choose the tower to be identified.

How to Choose the Tower. In the process of dynamic capacity-increase of transmission line, the tower that is most worthy of attention should be the point where the wire temperature measurement is the highest on the same line. So it is simple and direct to make the decision that the highest point of the wire temperature measurement will be chosen.

For a transmission line, in most of the time, the conductor temperature of the highest point is a single or a few points in the core of the city because of the heat island effect. Therefore, the above method is not only convenient, but also feasible.

Taking the above factors into account, we presented the measurement point selection scheme.

The temperature measurement value of all points (take the average value of the measurement of the temperature at the point) on the same line will be compared every one hour, and the measured point of the highest temperature will be chosen to be calculated, and the identification results are integrated as the identification result of this article.

Then we need to do some pre-processing of the micro meteorological monitoring data.

Pre-processing of the Micro Meteorological Data. In order to improve the identification accuracy, a multi set of micro meteorological data measuring device is needed in the same measuring point. For multiple measurements of the same physical quantity, the identification process is as follows. 
For example, the number of the environmental temperature monitoring device in tower $\mathrm{A}$ is $k$. In the $n-1$ time intervals, the environmental temperature measurement values of the number $a(1 \leq a \leq k)$ device are $t a_{1}, t a_{2}, t a_{3}, \ldots . ., t a_{n}$. And we let $t a_{i+1}=$ tave $_{i+1}$ if it meets $t a_{i+1}<(1-5 \%)\left(t a_{i}+t a_{i+2}\right) / 2$ or $t a_{i+1}>(1+5 \%)\left(t a_{i}+t a_{i+2}\right) / 2$, the tave , $_{\text {tave }}$, tave $_{3}, \ldots . .$, tave $_{n}$ here are the mean values of the measurement of devices during same time intervals. And we repeat the above steps until the completion of the delete operation.

Then we calculate the average values of the $k$ devices, and obtain the results $t A_{1}, t A_{2}, t A_{3}, \ldots \ldots, t A_{n}$ as the mean values of the measured temperature.

The processing method of the intensity of sunlight, wind speed and wind direction is similar to the above method.

Calculation of Conductor Temperature Estimation. There are three methods to calculate the estimated value of the wire temperature.

One method is to calculate after taking the measured micro meteorological data and current data into the simplified Morgan formula as Eq. 3. Using this calculation method, we consider that the conductor temperature at the measuring time point is in the steady state. However, in the actual process, it takes a few minutes or even tens of minutes for the wire temperature to reach the stable value when the micro meteorological conditions and current numerical changes. And during this period, the micro meteorological and current data is not static, that is, the conductor temperature is always in a transient process of change and doesn't reach the so-called steady state. Therefore, this estimation method can only be considered when the data sampling interval is large, and the transient process can not be accurately analyzed. In the case of small data sampling interval, this method isn't taken, because it does not take into account the transient process of temperature changes in the wire, and the result is not accurate enough.

The second method is to use the simplified transient formula to estimate. This method requires the data sampling interval to be as small as possible, so that we can use the measured micro meteorological and current data at the time point to instead of the sampling interval data, and will not produce a large error. Then, in the first time interval, we use the micro meteorological and current data as well as the initial value of the conductor temperature, to find the numerical solutions of the transient Eq. 2 by using the Runge-Kutta method. The final numerical solution is the conductor temperature estimation at the end of the time interval, as well as the initial value of the conductor temperature at the next time interval. This step cycle, the conductor temperature can be estimated at the end of each time interval. In this way, the transient process of conductor temperature change is considered, and the measured value of conductor temperature is not used when conducting wire temperature estimation. In theory, these estimates should be the most close to the actual value. However, the actual situation is that each time interval estimation results will have error, these errors are caused by the measurement error and calculation error, in a certain time interval, these errors are very small, can be ignored; but when the time span is very long, these errors will be accumulated step by step, will eventually make the estimated results deviate from the actual value. Therefore, we consider that in each time interval, we correct estimation results of the last step, and then use the revised results in the next step calculation. And this is the third method which is taken in this paper.

As mentioned above, the third method is based on the second methods to add the correction of the results of each step, so as to ensure that in a longer duration, and the estimated results are reasonable. The specific method is that the initial temperature of each time interval is changed to the average value of the estimated value of conductor temperature at the last time interval and current time interval conductor temperature measurement, and this can ensure that the estimated temperature of the wire is not too far away from the actual value. Specific estimation procedure is as follows.

Assuming the data sampling interval is $\Delta t$, the number of continuous sampling is $n(n \geq 2)$, if the number of the conductor temperature acquisition devices at a location is $m(m \geq 1)$. Then the 
continuous sampling length is $\Delta t \times(n-1)$, the temperature measured data a total of $m \times n$, set it as Eq. 4.

$$
T_{m}=\left[\begin{array}{cccc}
T m_{11} & T m_{12} & \mathrm{~L} & T m_{1 n} \\
T m_{21} & T m_{22} & \mathrm{~L} & T m_{2 n} \\
\mathrm{M} & \mathrm{M} & \mathrm{O} & \mathrm{M} \\
T m_{m 1} & T m_{m 2} & \mathrm{~L} & T m_{m n}
\end{array}\right] .
$$

And at each time point, the number of the wire temperature estimated value data is $m \times n$, set it as Eq. 5.

$$
T_{e}=\left[\begin{array}{cccc}
T e_{11} & T e_{12} & \mathrm{~L} & T e_{1 n} \\
T e_{21} & T e_{22} & \mathrm{~L} & T e_{2 n} \\
\mathrm{M} & \mathrm{M} & \mathrm{O} & \mathrm{M} \\
T e_{m 1} & T e_{m 2} & \mathrm{~L} & T e_{m n}
\end{array}\right] .
$$

The initial temperature value is $T m_{i 1}$, and we use the micro meteorological data and current data at the time point 1 , then take the initial temperature value and micro meteorological data and current data into the transient thermal stability Eq. 2, to find a numerical solution $\operatorname{Tex}_{i 1}$ of the transient equation after a time interval $\Delta t$ by using the Runge-Kutta method. And then take $\left(T e x_{i 1}+T m_{i 1}\right) / 2$ as the initial temperature value at the same time interval, to find a numerical solution $T e_{i 1}$ of the transient equation after a time interval $\Delta t$ by using the Runge-Kutta method.

We take the following method to calculate $T e_{i j}(j \geq 2)$ :

The initial temperature value is $\left(T e_{i(j-1)}+T m_{i(j-1)}\right) / 2$, and we use the micro meteorological data and current data at the time point $j$, then take the initial temperature value and micro meteorological data and current data into the transient thermal stability Eq. 2, to find a numerical solution $T e_{i j}$ of the transient equation at the time point $j$ by using the Runge-Kutta method.

Model and Calculation of Conductor Temperature Identification. After obtaining the estimated temperature $T_{e}$, the weighted sum of squares of the difference between the measured value and the estimated value is used as the objective function, and the least square optimization model can be constructed for the wire temperature identification as Eq. 6-8:

$$
\begin{aligned}
& \text { obj. } \min \sum_{j=1}^{n} W_{i j}\left(T e_{i j}-T m_{i j}\right)^{2}, i \in\{1,2,3, \ldots \ldots, m\}, \\
& \text { s.t. } \frac{d T e}{d t}=\frac{Q_{I}+Q_{S}-Q_{R}-Q_{C}}{m C},(1 \leq i \leq m, 1 \leq j \leq n), \\
& \quad\left|T e_{i j}-T e_{k j}\right|<T D i f f_{\text {max }},(1 \leq i \leq m, 1 \leq k \leq m, 1 \leq j \leq n) .
\end{aligned}
$$

In the objective function Eq. $6, W_{i j}$ is the weight of the corresponding measuring point, which can be set according to the accuracy of the measurement device or the measurement history of the measurement accuracy, etc.. The calculation methods of the heat and heat capacity in equality

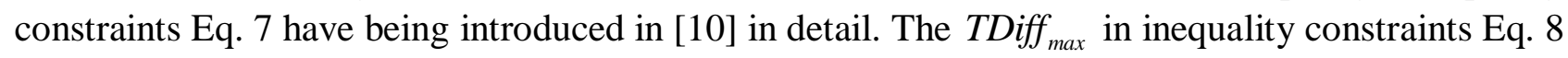
is a given threshold. 
Fig. 1 shows the results of the data identification, using the measuring data at the tower number 13 of XiaXiao2577 line for August 5th [11, 12]:

Data sampling start time is at 12:00:00a.m., end point is at 16:29:00p.m., the time of one interval is $\Delta t=60 \mathrm{~s}$, continuous sampling number is $n=270$ and continuous sampling for 269 , the number of conductor temperature acquisition devices is $m=2$, the number of measured temperature values is $2 \times 270$; set the weight of the measuring point to 1 , and the threshold $T$ Diff $f_{\max }$ to $0.5^{\circ} \mathrm{C}$.

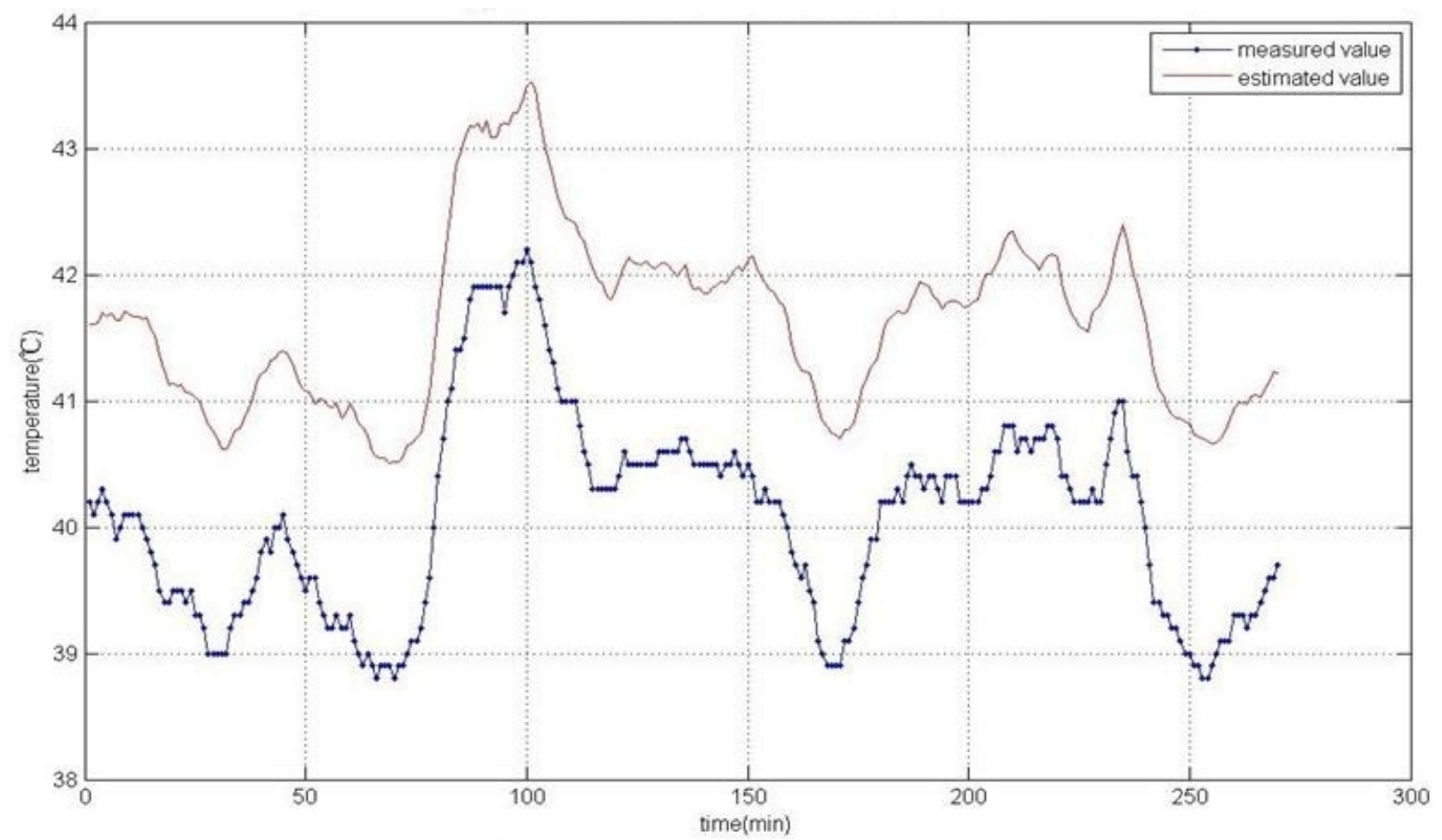

Fig.1 Temperature Identification Results for the XiaXiao2577 Line

\section{Identification Result Analysis}

It can be seen from the Fig. 1 that there is a constant difference between measured and estimated temperature of about $1.5^{\circ} \mathrm{C}$, this difference is caused by the error of the measuring and calculation, for the reason of the difference, there are two kinds of interpretation.

The first is the measurement error of the measured temperature. Considering the different temperature measuring devices produced by different manufacturers, the measurement results are different due to the different measurement methods and production processes. The measurement devices used in the project of this paper are all the same, so the results are not particularly comprehensive.

The second is the error of the calculated value of temperature. Because the parameters in the calculation formula of the traverse load are the experimental values which have been obtained by many experiments, there are many empirical parameters. Due to the change of the process and the change of the geography environment, these parameters can not be guaranteed. And the actual results show that the calculated parameters are in error, but in the actual project, it can be accepted.

In order to have a facilitate comparison, we move the estimated value of the curve down $1.5^{\circ} \mathrm{C}$ and get the Fig. 2, from this figure, we can be more intuitive to see that even for the details of the changes in the part, the results are more ideal identification. 


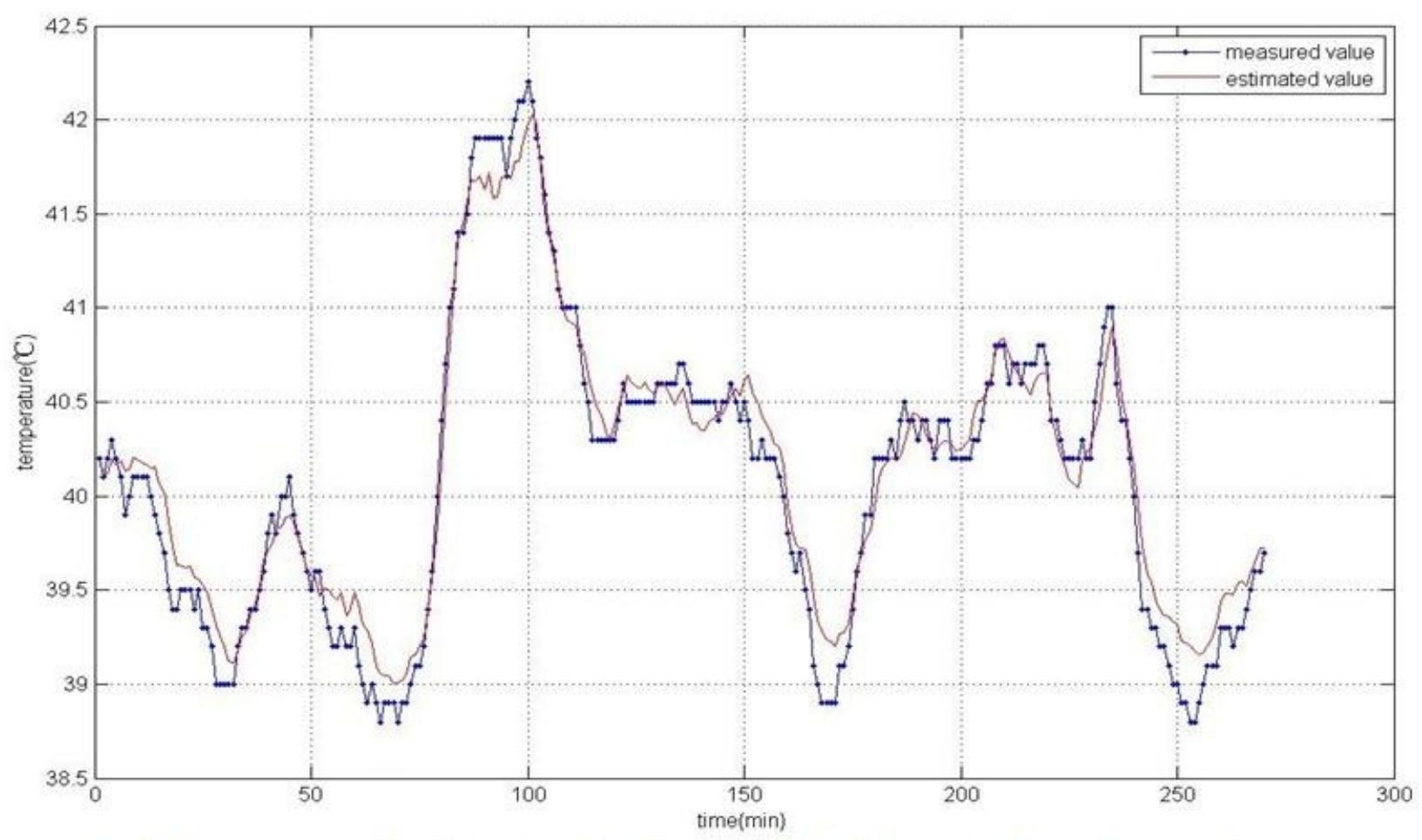

Fig.2 Temperature Identification Results for the XiaXiao2577 Line (downward curve)

\section{Conclusions}

Based on the detailed analysis of the influence factors of the dynamic capacity increasing of transmission line, a least square optimization model is proposed for the identification of the conductor temperature measurement in the process of dynamic capacity increasing. Then the identification method is tested by the measured data of the dynamic capacity increasing project. The results show that the method is feasible and worth to be further studied.

The identification method proposed in this paper has the following advantages.

Firstly, the identification method takes into account the transient process of temperature change, and the result is high reliability.

Secondly, Runge-Kutta method is used to solve the transient equation, that makes the method has good flexibility and faster computing speed.

Thirdly, the identification accuracy of the method will increase, if the time interval is smaller, or the number of the measuring devices at same point is more and the accuracy of measurement device is increase, which indicates that this method is more practical with the development of engineering technology.

\section{Acknowledgements}

This work was financially supported by the China Electric Power Research Institute (210003), Innovation Program of China Electric Power Research Institute (210003) and Hohai University (210098).

\section{References}

[1] DAVIS M.W: IEEE Transactions on Power Apparatus and Systems. Vol. 3 (1997), p. 803-809.

[2] DAVIS M.W: IEEE Transactions on Power Apparatus and Systems. Vol. 3 (1997), p. 810-825. 
[3] Q.P. Zhang, Z.Y. Qian: Transmission line capacity increasing technology (China Electric Power Press, Beijing 2010). In Chinese.

[4] The Ministry of electric power industry, electric power planning and Design Institute: Power system design manual (China Electric Power Press, Beijing 1998). In Chinese.

[5] L.J. Ren, G.H. Sheng, L.X. Li, Y Zheng and X.C. Jiang: Automation of Electric Power Systems. Vol. 30 (2006), p. 45-49. In Chinese.

[6] P. Yang, X.Y. Fang: East China Electric Power. Vol. 33 (2005), p. 11-14. In Chinese.

[7] Z.Q. Ye, H.P. Zhu: Power System Technology . Supplement Vol. 30 (2006), p. 258-263. In Chinese.

[8] Y.Z. Lin: Southern Power System Technology. Vol. 6 (2012), p. 23-27. In Chinese.

[9] Q.P. Zhang, Z.Y. Qian: Power System Technology. Vol. 29 (2005), p. 18-21. In Chinese.

[10] Y Dai, Y.C. Cheng, W.L. Zhong and J.D. Lin: Dynamic capacity increasing technology of high voltage overhead transmission lines (China Electric Power Press, Beijing 2013). In Chinese.

[11] DL/T 5092-1999, Technical Code For Designing 110kv 500kv Overhead Transmission Line (1999). In Chinese.

[12] GB/T 1979-1999, Round Wire Concentric Lay Overhead Electrical Stranded Conductors (1999). In Chinese. 